\title{
A quasi-experimental and Guided Social Media Intervention to Improve Mental Health Literacy Level of Urban School Adolescents in Ethiopia: A Detailed Study Protocol
}

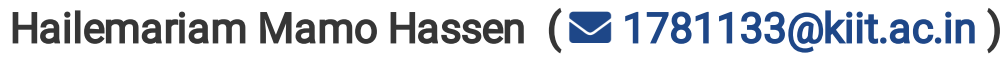

Dire Dawa University https://orcid.org/0000-0002-9584-2408

Manas Ranjan Behera

KIIT University

Pratap Kumar Jena

KIIT University

Sudhir Kumar Satpathy

KIIT University

Study protocol

Keywords: Mental Health Literacy, Quasi-experiment, School Adolescents, Guided Social Media Intervention

Posted Date: March 13th, 2020

DOI: https://doi.org/10.21203/rs.3.rs-17074/v1

License: (c) (1) This work is licensed under a Creative Commons Attribution 4.0 International License.

Read Full License 


\section{Abstract}

Background: Studies showed Ethiopian adolescents have lower knowledge and awareness about resilient and risk factors and preventive methods of mental health problems that increases mental health promotion and prevention gap. Addressing this gap in school settings using technology assisted mental health promotion platforms could help improve mental health literacy level. Therefore, evaluating effectiveness outcomes of guided social media intervention to improve mental health literacy of school adolescents is convenient issue.

Methods: Quantitative and quasi-experimental study will be used among urban school adolescent in Dire Dawa, Ethiopia. Following pretest measure among adolescents who have smart phones or social media access and age $>15$ years old, participants will be assigned by regression discontinuity design as good as random and then mental health literacy module will be delivered through face book/telegram group to be created for about four weeks and posttest will be done after lapse of a week. Control group will receive posts on effective academic studying skills. Adapted and tested questionnaire in convenient local languages along with English version will be used. Inferential statistics will be applied with significance level of $p<0.05$. Informed voluntary consent will be obtained from participants, their parents/guardians and school directors. Ethical approval is already obtained.

Discussion: Mental health promotion in school settings most importantly using technology assisted mental health promotion platforms supposed to be cost-effective, accessible, acceptable, adoptable, appropriate, feasible, reliable, scalable and sustainable approach helping tailor mental health literacy focused mental health first aid practices still deficient and new for Ethiopia. The study will test the hypothesis whether social media is effective channel of intervention in improving mental health literacy level of urban school adolescents.

\section{Background}

Globally, about $10-20 \%$ of children and adolescents have mental health problems[1,2]. Several studies depicted that most of mental health problems in adulthood essentially start to happen during adolescence[3,4]. A worldwide-pooled meta-analysis from all regions of the world from twenty seven countries reported that about $13.4 \%$ child and adolescent have mental disorders[5]. A meta-analysis study in sub-Saharan Africa including Ethiopia showed significant number of adolescents have psychopathological problems $(14.3 \% ; 13.6 \%-15.0 \%)$ and mental disorders $(19.8 \% ; 18.8 \%-20.7 \%)[6]$. Similarly, prevalence of common mental health problems among adolescents in Ethiopia was reported to be about $17-23 \%$ with substantial mental health disparities[7,8].

Empirical studies have shown that most of adolescents having mental health problems have usually limitation in help seeking and delay to get timely help[4,9-11]. A number of adolescents do not recognize how and when to get help for their mental health difficulties[12]. It is mainly because of low help-seeking intention and behaviour reflected from lack of recognition and knowledge about the nature of mental 
health problems, their symptoms and source of helps[4]. Therefore, adolescents have become among one of the most intervention population segments for multiple reasons. Firstly, adolescence is a critical physical, emotional and cognitive developmental period[13-15]. Secondly, basic cognitive, emotional physical and developmental processes shaping health-related behaviours and skills occurs during adolescence[16]. Thirdly, public health perspective for early intervention and prevention essentially help improve ability to understand health information[17] and assumed to assure later life with positive health outcomes [18]. Fourthly, mental health problems and chronic illness are growing among the adolescent population[19]. It has significant contribution for adolescents themselves and for community and future generation[20].

Mental health literacy is knowledge, belief, attitude and ability to recognize mental disorders, risk factors and causes, professional help available for treatment, help-seeking and self-help strategies, how to seek mental health information and intention and anti-stigma attitude to whom with mental health problems[21-23]. Low mental health literacy have become among substantial risk factors for modernday mental health problems becomes among the prior challenges in the global health efforts[24].

Technology has become vital for accessing health information and educational interventions[18]. Most importantly, social media has provided numerous opportunities and become an attractive platform for exchange of health information[25,26]. It promotes social connectedness among adolescents and youth and improving resilience and wellbeing[26]. Online technologies have become hugely integrated with adolescents' daily live activities[27]. Mainly, social media is among rapid and innovative advancements in participative internet communications offering opportunities for sharing health information and changing health behaviour[20].

Adolescents and young adults are the highest population portion to use social media compared to any age group[28] that allows adolescent users to create, share, and exchange information and ideas in virtual networks[29]. For that reason, interventions using online technology has become important and preferable methods overcoming multiple physical and logistic barriers and challenges[9]. It is helpful to reduce stigma providing low threshold access with better reach, eventually enabling individuals who face mental health problems to access and get help and even improving the help-seeking intention and behaviour of young adult and adolescents[30].

However, empirical data on level of mental health literacy and the impact and usefulness of school based mental health promotion platforms using social media are rare in developing countries[31,32]. In Ethiopia, there exists lack of evidences on effectiveness of technology assisted mental health promotions particularly on effectiveness of guided social media interventions. Therefore, the present study will test the hypothesis whether guided social media intervention is effective in improving mental health literacy level of urban school adolescents. Additionally, its acceptability, appropriateness and feasibility will be assessed as secondary objective.

\section{Methods/design}




\section{Study setting and study participants}

The present study will be conducted in Dire Dawa City, Ethiopia, which is trade collider of Eastern Ethiopia with diverse traditional practices, economic and socio-demographic features. It is the second largest and self-administrative city next to the capital, Addis Ababa found in the latitude of 9, $5833\left(934^{\prime} 59.988\right.$ " $\mathrm{N}$ ) and longitude of 41, $8667\left(4152^{\prime} 0.120^{\prime \prime} \mathrm{E}\right)[33]$. The source population will be urban regular school adolescent and youth students in Dire Dawa city from both public and private secondary schools.

- $\quad$ Inclusion and Exclusion Criteria

Urban school adolescents who are within age of 15 and above years and attending regular schooling will be included. These students will be from grade 9 to 12 level from public and private junior and secondary schools. Students those having smart phones or social media access will be included. Adolescents who have no smart phone and/or unable to access social media will be excluded.

\section{Participants' Incentive}

In addition to assumption of altruism from the study participants, motivational incentive will be provided to encourage participants that improve the recruitment and retention. Study participants will not receive direct financial payment for their participation. However, two palm top computers (tablets) will be prepared for participants to be drawn for a draw/lottery. Study participants who will be provided daily 45 MB internet package during intervention interaction. Additionally, one hundred Ethiopian birr mobile card will be provided for ten intervention participants who will be selected to coordinate active participation/involvement during intervention.

- $\quad$ Sample Size determination and procedures

Both the control and intervention group participants will be selected and assigned by using regression discontinuity design (RDD). Participants who will meet criteria will be assigned in both groups "as good as random" to intervention receiving group (group A) and the other as a non-equivalent control group (Group B). All socio-demographic characteristics like age, gender and grade level will be balanced. The sample size will be calculated by the following formula based on standard deviation of difference in difference and size effect (d) that is taken from similar study on mental health literacy intervention effectiveness[34].

Sample size $(n)=2 S D^{2}\left(Z_{\alpha / 2}+Z_{\beta}\right)^{2}$; for each intervention and control group.

$d^{2}$

$\mathrm{SD}=$ Standard deviation of the intervention group from a study $=0.9$

$Z_{a / 2}=Z_{0.05 / 2}=Z_{0.025}=1.96$ (From $Z$ table) at type 1error of $5 \%$ 
$Z_{\beta}=Z_{0.10}=1.282$ (From $Z$ table) at $90 \%$ power

$d=$ effect size $=$ difference between mean values a previous study on school adolescents $=0.3$

Sample size $(n)=\frac{2^{* 0.9^{2}(1.96+1.282)^{2}}}{0.3^{2}} ;$ for each intervention and control group.

$\mathrm{n}=113.65 \sim 114$ for each group

Insert here - Figure 1: Quasi-experimental study design showing study procedures developed by the authors

\section{Data Collection Methods}

Data collectors will be trained individuals who have at least basic understanding of health sciences and data collection skills; and who are able to speak the languages that the questionnaire is prepared (English, Amharic, Afaan Oromo and Somali). Providing the details about the nature of the study procedures; and then getting the participants and their parents consent as well as the school principals' consent in prepared written consent form, data collection will be done in schools as per the students' convenient time. The questionnaire will be provided for each participant who is willing and get his/her parents signed consent and he/she himself/herself do so.

\section{Intervention}

The intervention will focus on broad mental health awareness training and packages addressing a variety of specific mental health issues and conditions. Manual addressing mental health literacy contents is prepared as per African school mental health curriculum guide, Canadian and Australian school adolescents' mental health literacy delivery and mental health first aid training manuals [29,36-38].

The manual will be prepared in a way convenient to deliver on guided social media in plain English language in a simple and concise format with texts, figures and cases in vignettes. School adolescents recruited for intervention will be interconnected with each other forming social media particularly Facebook/Telegram group. Facebook and Telegram are preferred for intervention because they are mostly used among Ethiopian adolescents.

The intervention receiving group of school adolescents will get structured and interactive mental health literacy information and brainstorming scenarios for length of four weeks. They will discuss and exchange mental health information and locally traditional attitudes towards mental illness, stigma, and barriers for help-seeking and unhealthy mental health behaviours.

The control group of school adolescents will get interactively brainstorming information and tips about academic studying skills from a book entitled "Study skills handbook" [39] for same length of four weeks. 
They will discuss and exchange these topics. After one-week lapse of intervention data will be collected again from both control and intervention receiving groups.

- $\quad$ Intervention procedures and steps

Schools adolescents fulfilling the inclusion criteria will be assigned "as good as random" to intervention and control groups. They will be registered in Facebook/Telegram group to be created for this purpose immediately during selection for intervention and control groups after briefing detailed information about the study procedure and rubrics. The intervention activities sequentially include the following but not limited to:

1. Adapting mental health literacy module refined in contents and scope and to be posted in different forms on page of Facebook/Telegram.

2. Conducting pilot intervention to propose variance in the effect of the intervention and for situational testing to be improved in the main intervention.

3. Screening participants who fulfill inclusion criteria of the study.

4. Providing brief information about the study procedure and rubrics and will be requested to apply for participation.

5. Allocating and registering to Facebook/Telegram groups for intervention (group A) and the other for control (group B).

6. Selecting peer mentors from both groups who will facilitate intervention activities and as focal person to handle unforeseen situations.

7. Contents of the module will be posted every $72 \mathrm{hrs}$ ( 3 days) difference totally 8 session posts within the intervention time period of four weeks and remaining on week summary post.

8. Motivating and inspiring and monitoring students to interact, to comment, to ask and to answer questions for about four consecutive weeks.

9. Discussing and summarizing online at the end of every week for approximately 50 to 60 minutes for every 2 session posts on the topic(s) covered and briefing for next. This will be as booster sessions aiming at helping adolescents contextualize the contents into their daily life and conclusion of sessions. The intervention will consist of eight parts of the module.

Sessions posts will be in the form of texts, pictures, and short audio and video clips, testimonials with same contents of the prepared manual. Additionally, there will be interactive quizzes, exercises and content-oriented entertaining and motivational messages. Participant school adolescents will be encouraged to explore similar individualized mental health information based on their specific concerns and preference.

10. Same processes will happen to school adolescents from group B (control) but, different content of discussion that will be basically on academic studying skills. 
11. After four weeks, post-test data will be collected from both groups in the same way after a week lapse of intervention completion to assess its impact and effectiveness. Participants of the intervention group will also rate ( 1 = strongly disagree to $4=$ strongly agree) for their perception and agreement with statements about the intervention training in general to measure its acceptability, appropriateness, feasibility and satisfaction.

\section{Measures}

Self-administered questionnaires will be used in a supervised manner assisting those in the request for assistance. The Measurement tools are framed with cognitive skills focusing on individual knowledge, understanding, competence, and practice and perceived ratings with likert scale oriented to pencil and paper testing. These tools are adapted from previously tested questionnaires for their validity and reliability that will be also modified/adapted and revalidated further in Ethiopian settings to be contextualized for societal, cultural and sub-cultural suitability and sensitivity. These tools will have the following parts along with questions assessing socio-demographic characteristic.

\section{Primary outcome measures}

- Scale based mental health literacy questionnaire(MHLQ) and tested and validated for adolescent populations[22,40-48]

- Health seeking intention questionnaire[49]

\section{Secondary outcome measures}

- Other intervention outcome measures (acceptability appropriateness, feasibility)[50] and adapted satisfaction questionnaire to the social media health interventions(CSQ-I)[51]. These measurements will be used to assess the perspective and perception of adolescents about indicators of acceptability, appropriateness, feasibility and satisfaction of interventions as evidence for the social media intervention effectiveness outcomes.

\section{Statistical analyses}

- $\quad$ Effect Size

Impact and effectiveness of social media intervention on mental health literacy level of school adolescents will be analysed using effect size (Cohen's d value). Effect size is the magnitude of the mean score difference between intervention and control groups. The absolute effect size is the difference between the average/mean of mental health literacy score in the two groups. 
Similarly, Indirect effect of the intervention on mental health literacy intervention for changes in related outcomes such as generic health literacy level, health/help seeking behaviour, self-perceived health, and mental health level and healthy behavioural practices will be analyzed in similar ways and using paired and unpaired t-test.

- $\quad$ Single difference impact estimates and difference in difference:

It will done by taking difference of baseline (pre-test) score and end-line (post test score) and the score differences between the intervention group and the control group score difference.

The indicators of acceptability, appropriateness, feasibility and satisfaction of interventions as evidence for the social media intervention effectiveness outcomes will be analysed for each set cut off point.

\section{Organization, Quality Assurance, and Data Management}

Careful data collection procedures will be followed in face to face assisted and guided questioning approach that respondents will fill questionnaires in specific classrooms with proximate help and immediate briefing and clarification as necessary. Due efforts will be made to minimize systematic errors/bias giving proper care and emphasis during study design, sampling, data collection, analysis, interpretation, and conclusion. Biases that may be arising from analyzing, power/sample size, interpretation, reporting, presenting and during publication will get in each spot attention.

The utmost attempt will be done to reduce sources of bias and errors focusing on the following points throughout from design stage to conclusion on study findings.

1. A sequence of orientation and close discussion will be done with study participants so that response bias will be minimized.

2. Measurement tools will be already validated ones and will be adapted and tested by a pilot study on study settings for its reliability consideration(Cross-cultural adaptation (CCA) of the questionnaires)

3. Motivational and inspirational schemes will be prepared to increase the willingness of study participants and their concern on quality response and follow up.

4. Respondents will fill questionnaires in specific classrooms with intimacy and immediate briefing and clarification as necessary in face to face assisted and guided questionnaire approach.

5. Double checking in data recording/entry

6. A proper descriptive and inferential analysis will be done in standardized models

7. The analysis will be in an objective manner

8. Generally, extreme care and due attention will be given in every stage of the study.

The effect modifiers and confounding factors will be controlled by regression discontinuity design (RDD) (assigning eligibility criteria for intervention) and stratification of the data during analysis. In addition to 
the stated and assigned eligibility criteria for intervention. Urban adolescents' background and sociodemographic characteristics supposed to be effect modifiers or confounding factors will be considered for stratification analysis to control these effects and factors.

\section{Discussion}

The newly emerging technology create a health literate public advancing the understanding of the health risk factors and individuals ability to make decisions based on reliable health knowledge and understanding[52]. Adolescents and young adults are the highest population portion to use social media compared to any age group[28] that allows these adolescent users to create, share, and exchange information and ideas in virtual networks.

Despite of the higher school adolescents digitalized practices and usage of social media, studies on intervention effects of social media on adolescents' mental health literacy, help seeking intention and behaviour is limited in developing countries including Ethiopia. Schools are also an ideal setting promoting children and adolescents health[53]. Mental health promotion in school settings most importantly using technology assisted mental health promotion platforms supposed to be cost-effective, accessible, acceptable, adoptable, appropriate, feasible, reliable, scalable and sustainable approach helping tailor mental health literacy focused mental health first aid practices[20,54]. Social media provides opportunities for health information exchange $[25,26]$ promoting social connectedness in adolescents and youths[26]. Therefore, this interventions study using guided social media preferably will play role in advancing further intervention strategies overcoming physical and logistic barriers and challenges for adolescent mental health promotion in school settings[9].

\section{Limitation of the study}

It is quasi experiment and for its attributes, there are inherent differences in the intervention and control groups as the school adolescents, are located in distance and in different their existing schools and conditions. The social media literacy variation among the intervention receiving groups may influence the consistency of the degree of intervention effectiveness. Social media contamination is another anticipated limitation. Information privacy and information disclosure might fall in question during discussion although these will happen based on participants consent and willingness.

\section{Declarations}

\section{Ethical approval and consent to participate}

Ethical review was granted from KIIT University, Institute of Medical Sciences Institutional Ethics Committee (Ref. No. KIIT/KIMS/IEC/63/2019). The risk of being participating in this study is very minimal most importantly taking some amount times of the participants from their time. Informed, voluntary, written and signed consent will be obtained from each of the participant and their respective 
parents/guardians for students of age range 15-18 years. Students whose age eighteen and above years will be asked for their written consent to participate or not to participate in the study. Written consent will be obtained from School principals. The informed voluntary consent will be obtained ensuring the provision of comprehension information by their preferred language thoroughly describing the purpose, risks, benefits and their rights. Respect for anonymity and confidentiality of the participants' information will be maintained.

\section{Consent for publication}

Not applicable

\section{Availability of data and material}

Not applicable

\section{Competing interests}

The authors declare that they have no competing interests.

\section{Funding}

KIIT University and Ministry of Ethiopian Science and Higher education

\section{Authors' contributions}

HMM designed the study, analysed, interpreted data and wrote the manuscript. MRB, PKJ andSKS provided advisory, guidance and involved in framing the study protocol.

\section{Acknowledgements}

Not applicable

\section{Authors' information}

*HMM is a PhD Candidate, KIIT University, School of Public Health and Lecturer, Dire Dawa. University, Ethiopia

MRB and PKJ are Associate Professors in KIIT University School of Public Health

SKS is Professors and Director in KIIT School of Public Health, KIIT University 


\section{References}

[1]Ling J, Tay YF, Klainin-Yobas P. Mental health literacy levels. Arch Psychiatr Nurs 2018. doi:10.1016/j.apnu.2018.04.007.

[2]WHO. Adolescents: health risks and solutions: https://www.who.int/news-room/factsheets/detail/adolescents-health-risks-and-solutions 2018.

[3]David K, Park MJ, Mulye TP. The Mental Health of Adolescents: A National Profile, 2008. San Francisco: Natl. Adolesc. Health Inf. Cent. (NAHIC), Univ. Calif. 2008.

[4]Heike E. et al. The Mental Health of Adolescents: A National children and adolescents with StresSOS using online or face-to-face interventions: study protocol for a randomized controlled trial within the ProHEAD Consortium. Trials 2019;20:94. doi:10.1186/s13063-018-3157-7.

[5]Polanczyk G V., Salum GA, Sugaya LS, Caye A, Rohde LA. Annual research review: A meta-analysis of the worldwide prevalence of mental disorders in children and adolescents. J Child Psychol Psychiatry Allied Discip 2015;56:345-65. doi:10.1111/jcpp.12381.

[6]Cortina M, Sodha A, Fazel M, Ramchandani PG. Prevalence of Child Mental Health Problems in SubSaharan Africa _ Global Health _ JAMA Pediatrics _ The JAMA Network. Arch Pediatr Adolesc Med 2012;166:276-81.

[7]Zeleke WA, Nichols LM, Wondie Y. Mental Health in Ethiopia: an Exploratory Study of Counseling Alignment with Culture. Int J Adv Couns 2019. doi:10.1007/s10447-018-9368-5.

[8]CSA and UNICEF Ethiopia. Multidimensional Child Deprivation in Ethiopia. UNON Publ Serv Sect Nairobi-2018.

[9]Divin N. Help-Seeking Measures and Their Use in Adolescents: A Systematic Review Help-Seeking Measures and Their Use in Adolescents: A Systematic Review. Adolesc Res Rev 2018;0:0. doi:10.1007/s40894-017-0078-8.

[10]Tully LA, Hawes DJ, Doyle FL, Sawyer MG, Dadds MR. A national child mental health literacy initiative is needed to reduce childhood mental health disorders. Aust New Zeal J Psychiatry 2019;00:000486741882144. doi:10.1177/0004867418821440.

[11]Cornally N, Mccarthy G. Help-seeking behaviour: A concept analysis. Int J Nurs Pract 2011;17:280-8. doi:10.1111/j.1440-172X.2011.01936.x.

[12]Nagata JM, Ferguson BJ, Ross DA. Research Priorities for Eight Areas of Adolescent Health in Lowand Middle-Income Countries. J Adolesc Heal 2016;59:50-60. doi:10.1016/j.jadohealth.2016.03.016. 
[13]Patton GC, Olsson CA, Skirbekk V, Saffery R, Wlodek ME, Azzopardi PS, et al. Adolescence and the next generation. Nature 2018;554:458-66. doi:10.1038/nature25759.

[14]La Maison C, Munhoz TN, Santos IS, Anselmi L, Barros FC, Matijasevich A. Prevalence and risk factors of psychiatric disorders in early adolescence: 2004 Pelotas (Brazil) birth cohort. Soc Psychiatry Psychiatr Epidemiol 2018;53:685-97. doi:10.1007/s00127-018-1516-z.

[15]Mills KL. Social development in adolescence: brain and behavioural changes. PQDT - UK Irel 2015.

[16]Bröder J, Okan O, Bauer U, Bruland D, Schlupp S, Bollweg TM, et al. 05. Health literacy in childhood and youth: A systematic review of definitions and models. BMC Public Health 2017;17:1-25. doi:10.1186/s12889-017-4267-y.

[17]Johnson RL. Pathways to adolescent health: Early intervention. J Adolesc Heal 2002;31:240-50.

[18]Manganello JA. Health literacy and adolescents: A framework and agenda for future research. Health Educ Res 2008;23:840-7. doi:10.1093/her/cym069.

[19]Fok MSMM, Wong TKSS. What does health literacy mean to children? Contemp Nurse a J Aust Nurs Prof 2002;13:249-58. doi:10.5172/conu.13.2-3.249.

[20]Freeman JL, Caldwell PHY, Med B, Bennett PA, Sc BAL, Scott KM. How Adolescents Search for and Appraise Online Health Information: 2018. doi:10.1016/j.jpeds.2017.11.031.

[21]Claire M Kelly, Anthony F Jorm, Anne Marie Wright. Improving mental health literacy as a strategy. Med J Aust Med J Aust 2007;187:25-729.

[22]Kelly CM, Jorm AF, Wright A. Improving mental health literacy as a strategy to facilitate early intervention for mental disorders Claire 2007;187:1-5.

[23]Jorm AF. Why We Need the Concept of "Mental Health Literacy." Health Commun 2015;30:1166-8. doi:10.1080/10410236.2015.1037423.

[24]Dodson S, Good S OR. Health Literacy Toolkit for low- and middle-income countries: a series of information sheets to empower communities and health systems. New Delhi: World: 2015.

[25]Lin HC, Chang CM. What motivates health information exchange in social media? The roles of the social cognitive theory and perceived interactivity. Inf Manag 2018. doi:10.1016/j.im.2018.03.006.

[26]Szeftel R, Schlozman S, Bostic JQ, Piacentini E, Tabor-Fumark K, Feldmeier M, et al. Functional Assessment of Social Media in Child and Adolescent Psychiatry. Child Adolesc. Psychiatry Media, 2018. doi:10.1016/b978-0-323-54854-0.00008-4.

[27]Mitchell C, McMillan B, Hagan T. Mental health help-seeking behaviours in young adults. Br J Gen Pract 2017;67:8-9. doi:10.3399/bjgp17X688453.

Page 12/15 
[28]Hausmann JS, Touloumtzis C, White MT, Colbert JA, Gooding HC. Adolescent and Young Adult Use of Social Media for Health and Its Implications. J Adolesc Heal 2017;60:714-9.

doi:10.1016/j.jadohealth.2016.12.025.

[29]Kitchener BA, Jorm AF. Mental health first aid: An international programme for early intervention. Early Interv Psychiatry 2008;2:55-61. doi:10.1111/j.1751-7893.2007.00056.x.

[30]Kaess M, Ritter S, Lustig S, Bauer S, Becker K, Eschenbeck H, et al. Promoting Help-seeking using Etechnology for ADolescents with mental health problems: study protocol for a randomized controlled trial within the ProHEAD Consortium. Trials 2019;20:94.doi:10.1186/s13063-018-3157-7.

[31]Mulisa F, Getahun DA. Perceived Benefits and Risks of Social Media: Ethiopian Secondary School Students' Perspectives. J Technol Behav Sci 2018. doi:10.1007/s41347-018-0062-6.

[32]Kim S-Y, Ball K, Chow CK, Liu Y, Chen S, Redfern J, et al. The role of social media in preventing and managing non-communicable diseases in low-and-middle income countries: Hope or Hype? Heal Policy Technol 2019. doi:10.1016/j.hlpt.2019.01.001.

[33]CSA. Ethiopian Household Consumption: Results for Dire Dawa Administration, The Federal Democratic Republic Of Ethiopia: http://www.csa.gov.et/ehioinfo-internal?download = 859:hce-2016statistical-report-dire-dawa\&start $=5$. Cent Stat Agency 2018.

[34]Lo K, Gupta T, Keating JL. Interventions to Promote Mental Health Literacy in University Students and Their Clinical Educators. A Systematic Review of Randomised Control Trials. Heal Prof Educ 2018;4:16175. doi:10.1016/j.hpe.2017.08.001.

[35]GCYDCA. African School Mental Health Curriculum: Understanding Mental Health and Mental Illness: Guid Couns Youth Dev Cent Africa 2012.

[36]Stan Kutcher and Yifeng Wei of. Mental Health \& High School Curriculum Guide 2015.

[37]Kutcher S, Bagnell A, Wei Y. Mental Health Literacy in Secondary Schools. A Canadian Approach. Child Adolesc Psychiatr Clin N Am 2015;24:233-44. doi:10.1016/j.chc.2014.11.007.

[38]S. K, Y. W, C. M. Successful application of a Canadian mental health curriculum resource by usual classroom teachers in significantly and sustainably improving student mental health literacy. Can J Psychiatry 2015;60:580-6.

[39]Cottrell S. The Study Skills Handbook. Fourth Edi. PalgraveMacmillan inthe; 2013.

[40]0'Connor M, Casey L, Clough B. Measuring mental health literacy-a review of scale-based measures. J Ment Heal 2014;23:197-204. doi:10.3109/09638237.2014.910646. 
[41]Campos L, Dias P, Palha F, Duarte A, Veiga E. Development and psychometric properties of a new questionnaire for assessing mental health literacy in young people. Univ Psychol 2016;15:61-72. doi:10.11144/Javeriana.upsy15-2.dppq.

[42]Bale J, Grové C, Costello S. Mental Health \& Prevention A narrative literature review of child-focused mental health literacy attributes and scales 2018;12:26-35. doi:10.1016/j.mhp.2018.09.003.

[43]Lam LT. Mental health literacy and mental health status in adolescents: a population-based survey 2014:1-8.

[44]Dias P, Campos L, Almeida H, Palha F. Mental health literacy in young adults: Adaptation and psychometric properties of the mental health literacy questionnaire. Int $\mathrm{J}$ Environ Res Public Health 2018;15. doi:10.3390/ijerph15071318.

[45]Wei Y, McGrath PJ, Hayden J, Kutcher S. Mental health literacy measures evaluating knowledge, attitudes and help-seeking: scoping review. BMC Psychiatry 2015;15. doi:10.1186/s12888-015-0681-9.

[46]Sciences B. Mental health promotion in school context-Validation of the ES ' COOL scale for teachers 2018.

[47]Bjørnsen HN, Eilertsen MEB, Ringdal R, Espnes GA, Moksnes UK. Positive mental health literacy: Development and validation of a measure among Norwegian adolescents. BMC Public Health 2017;17:1-10. doi:10.1186/s12889-017-4733-6.

[48]Reilly MO, Svirydzenka N, Adams S, Dogra N. Review of mental health promotion interventions in schools. Soc Psychiatry Psychiatr Epidemiol 2018;53:647-62. doi:10.1007/s00127-018-1530-1.

[49]Divin N, Harper P, Curran E, Corry D, Leavey G. Help-Seeking Measures and Their Use in Adolescents: Systematic Review. Adolesc Res Rev 2018;3:113-22.doi:10.1007/s40894-017-0078-8.

[50]Saracutu M, Edwards DJ, Davies H, Rance J. Protocol for a feasibility and acceptability study using a brief ACT-based intervention for people from Southwest Wales who live with persistent pain. BMJ Open 2018;8:1-9. doi:10.1136/bmjopen-2018-021866.

[51]Bo L, Lehr D, Reis D, Vis C, Riper H, Berking M, et al. Reliability and validity of assessing user satisfaction with web-based health interventions. J Med Internet Res 2016;18:1-13. doi:10.2196/jmir.5952.

[52]Ratzan SC. Health literacy: Communication for the public good. Health Promot Int 2001;16:207-14. doi:10.1093/heapro/16.2.207.

[53]Mcluckie A, Kutcher S, Wei Y, Weaver C. Sustained improvements in students' mental health literacy with use of a mental health curriculum in Canadian schools. BMC Psychiatry 2014;14:1-6. doi:10.1186/s12888-014-0379-4. 
[54]Korda H, Itani Z. Harnessing Social Media for Health Promotion and Behavior Change. Health Promot Pract 2013;14:15-23. doi:10.1177/1524839911405850.

\section{Figures}

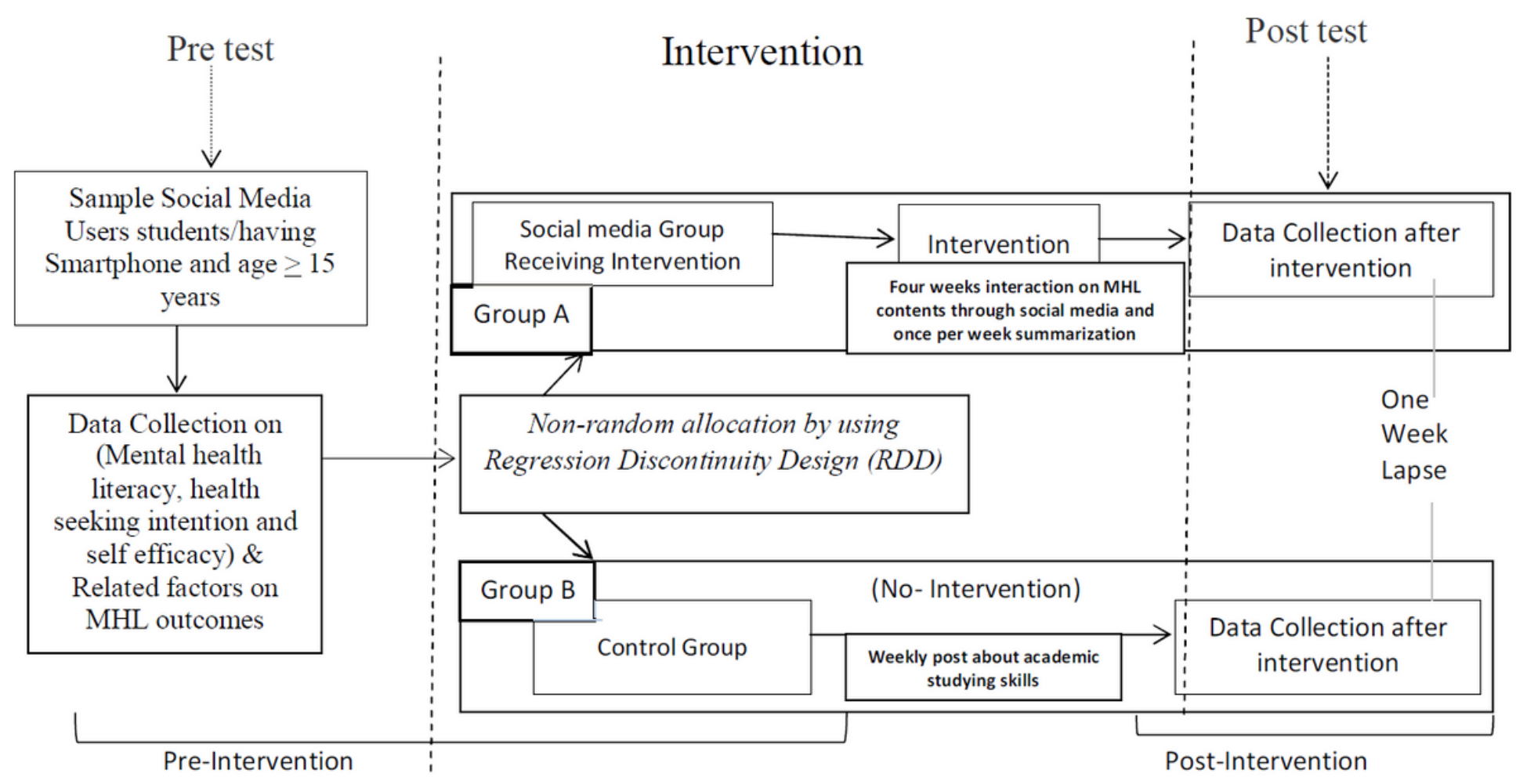

Figure 1

Quasi-experimental study design showing study procedures developed by the authors 